\title{
Procesos formativos durante el ejercicio docente: un estudio desde las creencias de profesores universitarios
}

\section{Training processes throughout teaching performance: a study from the beliefs of university professors}

\author{
Josefina Bailey-Moreno \\ Tecnológico de Monterrey, México \\ ORCID: https://orcid.org/0000-0003-1859-7629 \\ Manuel Flores-Fahara \\ Tecnológico de Monterrey, México \\ ORCID: https://orcid.org/0000-0001-9282-8750
}

Received 03-24-20 Revised 05-30-20 Accepted 07-07-20 On line 12-04-20

*Correspondence

Email: josefina.bailey@tec.mx
Cite as:

Bailey-Moreno, J., \& Flores-Fahara, M. (2020). Procesos formativos durante el ejercicio docente: un estudio desde las creencias de profesores universitarios. Propósitos y Representaciones, 8(3), e527. doi: http://dx.doi.org/10.20511/pyr2020.v8n3.490 


\section{Resumen}

Este estudio se propuso conocer cómo contribuyen los procesos formativos de profesores en ejercicio, en la construcción de creencias acerca de la enseñanza en la universidad. Con base en la metodología cualitativa y enfoque de teoría fundamentada se seleccionaron profesores de universidades públicas y privadas, mediante el muestreo teórico. Se realizaron entrevistas a profundidad para colectar datos y se codificaron analíticamente. Entre los resultados destacan las creencias que provienen de la capacitación institucional de tipo normativa, la cual enfatiza una enseñanza funcional, en técnicas didácticas, uso de tecnología e innovación, excluyendo la capacitación en el conocimiento de la disciplina que enseñan. Así también se encontró que los profesores llevan a cabo por iniciativa personal procesos de autoformación, para la actualización en conocimientos científicos, y desarrollan sus prácticas enseñando con estrategias que ellos mismos utilizan para aprender, porque creen en sus propios procesos de aprendizaje como una fuente valiosa y creíble para conducir la enseñanza.

Palabras clave: Creencia; Formación de docentes; Enseñanza superior; Profesor de universidad.

\section{Sumary}

This study aims to know how the training processes of practicing teachers contribute to the construction of beliefs about teaching at the university. From a qualitative methodology with a grounded theory approach, professors from public and private universities were selected through theoretical sampling. In-depth interviews were conducted to collect data which were analytically coded. Among the results it was found that the teachers' beliefs come from institutional training of a normative type which emphasizes a functional teaching in didactic techniques, use of technology and innovation, excluding training in knowledge of the discipline they teach. Thus, it was also found that teachers carry out self-training processes on a personal initiative for updating scientific knowledge and develop their practices teaching with strategies that they themselves use to learn, because they believe in their own learning processes as a valuable source and credible to conduct teaching.

Keywords: Beliefs; Teacher training; Higher education; University professor.

\section{Introducción}

Los procesos formativos en los que participan los docentes universitarios durante el ejercicio docente son fundamentales, ya que el conocimiento de la disciplina que enseñan, así como sus habilidades didácticas inciden directamente en el desarrollo de la enseñanza. Aún, hoy en día la investigación educativa destaca, por un lado, las críticas respecto a la manera instrumental en la que se llevan a cabo los procesos institucionales de formación docente, las prácticas estandarizadas, la función de técnicos e implementadores curriculares. Por otro lado, se insiste en que el aprendizaje y desarrollo docente se beneficia cuando estos participan en redes de colaboración, investigan y analizan su propia práctica, aprenden durante su trayectoria profesional en un continuo proceso formativo y participan activamente en las decisiones institucionales (Van Waes, De Mayer, Moolenaar Van Petergem y Van den Bossche, 2018).

Esta investigación se interesó por estudiar la manera en la que los profesores universitarios llevan a cabo procesos de formación durante el ejercicio docente, se abordó desde sus creencias, respecto a qué aprenden y cómo lo aprenden. Lo valioso de este acercamiento al pensamiento del profesor 
es que las creencias son conceptualizadas como conocimiento creído resultado de un proceso de validación de las fuentes o procedencia de dichas creencias. Adicional a las creencias declaradas por los docentes en los procesos de capacitación formal que ofrece la universidad, en este estudio se encontró que los profesores llevan a cabo una auto formación al tener iniciativas personales en la búsqueda de conocimiento y estrategias para conducir la enseñanza, un hallazgo interesante y no documentado en los estudios acerca de las fuentes de creencias: sus propios procesos de aprendizaje. Cabe señalar que este estudio forma parte de un proyecto más amplio denominado "Creencias de profesores acerca de la enseñanza en la universidad" llevado a cabo en el año 2018.

\section{Formación de docentes universitarios.}

Dado que, la función primordial de la Universidad en el siglo XXI es promover la educación humanista e integral, el desarrollo de actitudes y solución de problemas, así como la generación de conocimiento (De la Torre, 2013; Pérez y Castaño, 2016), el aprendizaje y formación de los docentes es crucial, ya que ellos a través de la práctica educativa son quienes pudieran ser coparticipes en el desarrollo de dichas funciones.

Sin embargo, se sabe que, en el contexto global los currículos se han ido adaptando a los requisitos del mercado y a políticas gubernamentales con la intención de orientar la enseñanza hacia prácticas basadas el uso de técnicas y herramientas didácticas, con énfasis en el desarrollo de competencias, priorizando los resultados de aprendizaje y la gestión empresarial (Day y Qing Gu, 2012; Hargreaves y Shirley, 2012). Debido a lo anterior, es imprescindible que la formación docente favorezca su pensamiento crítico y plural, el conocimiento pedagógico, así como habilidades para convivir en y para la democracia, con el fin de aminorar los efectos negativos producidos por el sistema de mercado y sus intentos de reducir la pedagogía a un conjunto de herramientas y a estimar el conocimiento por su utilidad, lo cual ejerce una baja valoración del desarrollo profesional (De la Torre, 2013; Gimeno, 2013; Giroux, 2013; Jarvis, 2013; Morin, 2014; Ornelas, 2009; Rué Domingo, 2015; Torres, 2007).

Los profesores universitarios son pieza clave para impulsar y alcanzar la educación para el desarrollo sostenible en la práctica (EDS, UNESCO, 2017). La EDS, vigente hoy en día, básicamente tiene como rasgos el aprendizaje a lo largo de la vida y calidad de vida para todos. En este sentido, la UNESCO (2017) recomienda que los profesores universitarios deben participar en la vida intelectual de las facultades e instituciones y de la comunidad en general; analizar los fundamentos en los que se basa la EDS; llevar a cabo investigación educativa; debatir críticamente asuntos complejos; establecer redes entre las facultades y disciplinas para examinar asuntos que plantean las dimensiones socio-económicas.

Day y Qing Gu (2012) señalan que estas redes de colaboración docente deben trabajar durante tiempos extensos ya que su creación lleva tiempo y se debe buscar un cambio sistémico con apoyo de los directores para crear ambientes participativos para la revisión de la práctica compartida. La UNESCO (2017) en el Informe de Seguimiento de Educación en el Mundo (Informe GEM, 2016, continuación del Informe de Seguimiento de Educación para Todos, 20012015) reporta que el aprendizaje y el bienestar laboral de los profesores es más alto cuando tienen la oportunidad de participar en la toma de decisiones de la escuela, colaborar con compañeros y recibir opiniones útiles de sus supervisores. 
Lo anterior refuerza la idea de que fortalecer la participación de los profesores puede facilitar el aprendizaje reflexivo para detectar y resolver problemas de sostenibilidad. La pedagogía crítica rechaza el papel del docente como transmisor y ejecutor de las ideas de otros, desde esta perspectiva el docente como intelectual comprometido, debía ser un investigador crítico y práctico reflexivo en la comprensión y transformación de la realidad (Carbonell, 2015; Giroux, 2013). Así también este rechazo a las prácticas estandarizadas, obedece a la negación de reducir el currículum al diseño de objetivos y programas, tareas docentes que, aunque muy importantes no resuelven el tema de la complejidad curricular (Carbonell, 2015; Díaz Barriga, 2016; Giroux, 2013).

Fullan, Rincón-Gallardo y Hargreaves (2015) desarrollan el concepto de capital profesional de los docentes para explicar cómo el aprendizaje individual y colectivo debía ser el eje en una nueva forma de rendición de cuentas basada en un sistema de toma de decisiones conjuntas. Así también indican que para que este sistema sea efectivo, los administradores y responsables de políticas educativas deben proporcionar las condiciones para el aprendizaje social, lo cual puede ser logrado a través de a) impulsar el aprendizaje continuo por medio de redes de docentes para el desarrollo de prácticas de enseñanza y el liderazgo, b) llevar a cabo investigación para resolver problemas complejos de práctica, y c) crear espacios para profesores y líderes educativos para identificar procesos institucionales y prácticas que limitan su trabajo y discutir soluciones para eliminar esas limitaciones.

\section{Creencias de profesores.}

Teorías y estudios acerca de la cognición de profesores (paradigma del pensamiento del profesor) han documentado que la riqueza de las creencias de maestros radica en que les proporcionan significado a las actividades que realizan en la escuela y el aula, ayudan a definir las tareas de enseñanza y a tomar decisiones, por consiguiente aportan a los maestros en su práctica, elementos de estructura, orden, dirección y valores compartidos (Bingimlas y Hanrahan, 2009; Ertmer, 2006; Luft y Roehring, 2007; Mansour, 2013).

Los teóricos clásicos de esta línea de indagación (Ernest, 1988; Kagan, 1992; Nespor, 1987 y Pajares, 1992) reconocen que la creencia es un constructo psicológico complejo, fácilmente confundible con otras construcciones del pensamiento como teorías implícitas, representaciones, actitudes u opiniones. Para su clarificación conceptual, resulta prudente explicar el significado de creencia desde esta perspectiva teórica, entendida esencialmente como conocimiento creído. En la figura 1 se esquematiza el proceso mediante el cual se llega a creer como resultado de someter el conocimiento a criterios de validez. Cabe señalar que en esta esquematización se representa de lado derecho el proceso de validación del conocimiento declarativo (saber conceptual), mientras que los aspectos representados bajo el criterio de aplicabilidad representan la validación del conocimiento procedimental (saber hacer). Esta manera de esquematizar obedece a fines de clarificación conceptual del término, lo cual no pretende reducir la complejidad de su funcionamiento. 


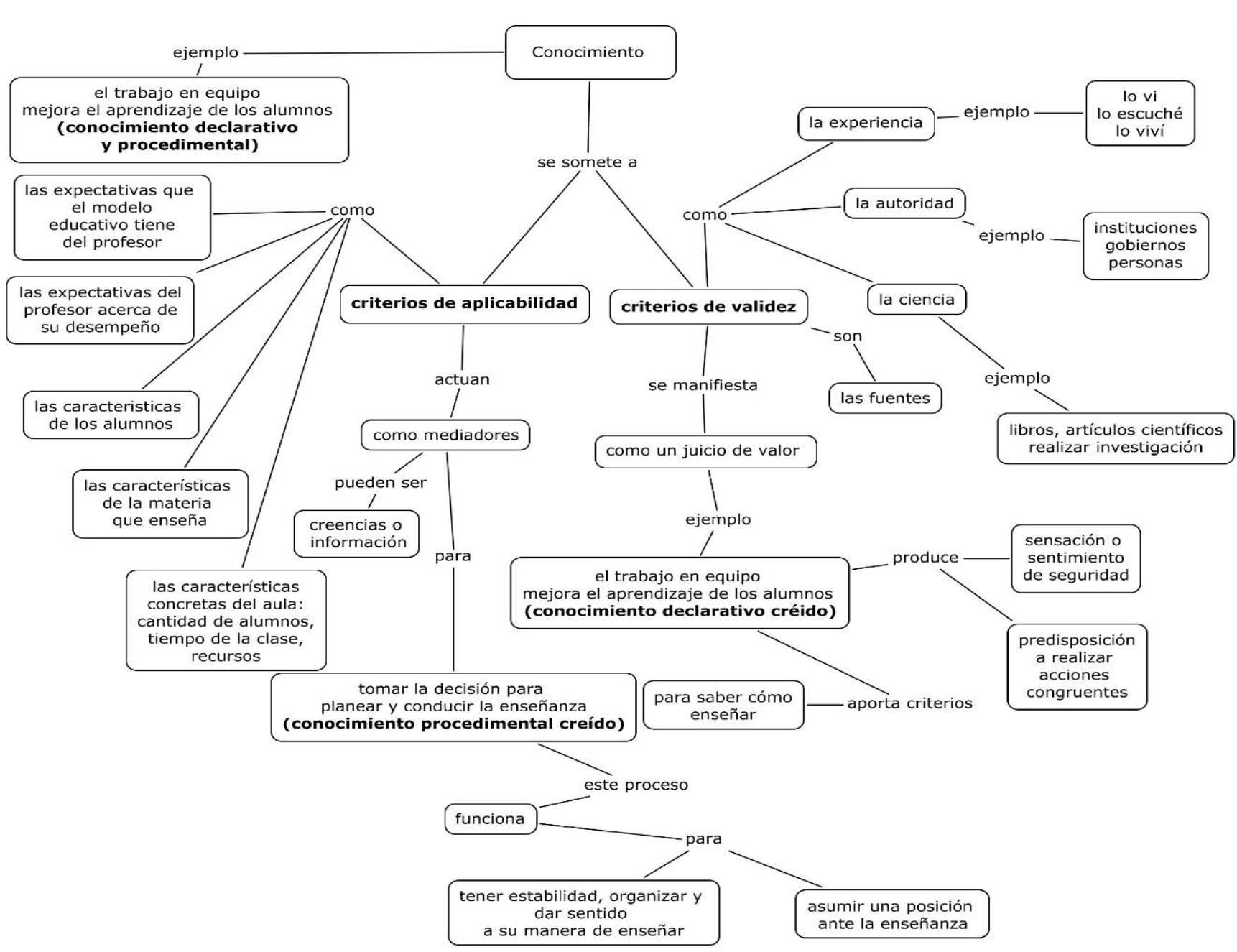

Figura 1. Proceso mediante el cual el conocimiento adquirido es creído

Fuente: elaboración propia

Para explicar cómo el conocimiento declarativo (saber) llega a ser creído, se recurre a Pajares (1992) quien retomó los modos de elección de creencias en Lewis (1990, 2002), al señalar que el conocimiento declarativo requiere creer en la autoridad de su origen, en la ciencia y en la experiencia. De este modo, las personas creen al considerar la validez de la fuente de información, por ejemplo, creer lo que se lee sobre ciencia en un libro, al considerar los libros como fuente válida de información.

Por criterio de validez se entiende al proceso por el cual el conocimiento una vez que ha sido adquirido (aprendido) se somete a criterios de validez para ser creído, esto se manifiesta como un juicio de valor, es decir las personas realizan un juicio o valoración al conocimiento, este proceso forma parte del funcionamiento cognitivo y su finalidad es proporcionar una estructura o marco de referencia que da significado a los sucesos y a las acciones que se realizan. Lewis (2002) estableció seis fuentes o criterios por los cuales las personas atribuyen validez al conocimiento: la experiencia, la autoridad, la ciencia, la deducción, la intuición y la fe.

Lewis (2002) precisó que para creer en un determinado conocimiento también se necesita creer en la fuente, por ejemplo, en la figura 1 el que un profesor crea que el trabajo en equipo mejora el aprendizaje supone que este profesor lo cree ya sea porque experimentó personalmente una mejora en su propio aprendizaje cuando fue estudiante (la experiencia), o cree porque lo leyó 
en un libro de pedagogía (la ciencia). Estos criterios de validez son lo que diversos investigadores han documentado como la función evaluativa de la creencia, la cual produce la sensación de seguridad en lo creído y por consiguiente, la idea de certeza y la predisposición a actuar en congruencia, este proceso explica también que la distinción entre conocimiento y creencia consiste en la valoración que se realiza al primero (Bingimlas y Hanrahan, 2009; Ertmer, 2006; Luft y Roehring, 2007; Mansour, 2013; Nespor, 1987; Pajares, 1992).

Respecto al criterio de aplicabilidad -el cual se denominó de ese modo en esta investigación- se utiliza para explicar el proceso mediante el cual el conocimiento creído llega a concretarse en acciones en la práctica educativa. En este sentido para saber qué hacer (qué procedimientos seguir) el profesor toma en cuenta las características institucionales, de los alumnos, de la idea de sí mismo como docente, del contenido de la materia que imparte, la cantidad de contenido a enseñar y otras características concretas del aula como el tiempo y los recursos disponibles para la enseñanza. Estas circunstancias actúan como mediadores para tomar decisiones en la planeación y conducción de la enseñanza. Siguiendo el mismo ejemplo del profesor que cree que el trabajo en equipo mejora el aprendizaje, al considerar estos mediadores puede decidir entre varias opciones, pudiera llegar a pensar que los estudiantes no saben trabajar en equipo porque se dividen el trabajo y decidir no implementar este tipo de estrategia en su enseñanza, o puede optar por implementarlo dado que una de las expectativas que la institución tiene de él como profesor es fomentar la colaboración entre los estudiantes.

Este modo de funcionamiento de la creencia complejo y dinámico conduce inevitablemente a la explicación de las relaciones existentes entre las creencias que conforman un sistema, el cual fue desarrollado inicialmente por Rokeach en 1968, este autor representó el sistema de creencias como un modelo central-periférico en el cual se encuentran todas las creencias que una persona tiene. En el centro se representan las creencias acerca de lo que la persona cree de sí mismo, todas las demás creencias tendrán una importancia determinada en la medida en que sean significativas o tengan un sentido para el yo (sí mismo). De este modo, las creencias que un profesor tiene acerca de la enseñanza son influidas por las creencias que tiene sobre sí mismo, sus fortalezas, áreas débiles en su desempeño y sus responsabilidades. Así también, las creencias que sostienen los maestros acerca del contenido de la disciplina que enseñan les informan la manera de organizarlo. Las creencias sobre los estudiantes, incluyen lo que significa ser un estudiante y la manera en la que los estudiantes deben relacionarse con los profesores. A su vez, todas estas creencias influyen en la planeación que realizan de la enseñanza y las prácticas que llevan a cabo en el aula, así como el tipo de interacciones con los estudiantes (Bingimlas y Hanrahan, 2009; Ernest, 1988; Ertmer, 2006; Luft y Roehring, 2007; Mansour, 2013; Murphy y Mason, 2012; Nespor, 1987 y Pajares, 1992).

Los estudios señalan que las creencias acerca de la enseñanza se adquieren (aprenden) en tres tipos de espacios educativos: 1) la educación formal cuando se fue estudiante, 2) la educación en las escuelas para futuros profesores y, 3) la capacitación durante el ejercicio docente. Así también indican que las creencias formadas cuando se fue estudiante pueden prevalecer y que para aprender nuevas creencias acerca de la enseñanza, la capacitación debe provocan vivir la experiencia de nuevas formas de enseñanza, de tal modo que para facilitar en los maestros el cambio de creencias y puedan desarrollar nuevas prácticas es importante que prevalezca en la capacitación un ambiente de cuidado y confianza en el cual los maestros estén dispuestos reflexionar sobre sus creencias (Baptista, Freire y Freire, 2020; Ertmer. 2006; Mansour, 2009; Nesport, 1987; Pajares, 1992 y Woolfolk, Davis y Pape, 2012). 
Como en la mayoría de las universidades no es requisito que los profesores tengan una educación formal como docentes (Montes y Suarez, 2016; Caballero, 2013) de acuerdo con los hallazgos teóricos y empíricos, se esperaría que los profesores enseñasen de acuerdo a las creencias formadas cuando fueron estudiantes y las aprendidas durante los cursos de capacitación (Solis, 2016). Como el conjunto de creencias que un profesor tiene han sido construidas (aprendidas) durante la trayectoria personal y profesional, en esta investigación se adoptó la conceptualización de procesos formativos a partir de la noción de desarrollo profesional propuesto por Imbernón (2002) como el conjunto de aspectos que posibilitan o impiden el progreso en el ejercicio de la profesión, tales como la capacitación que realizan los profesores y las condiciones de trabajo.

Pasillas (2011) señala que, aunque la enseñanza universitaria está enmarcada en el currículum como marco pedagógico, los docentes toman decisiones durante el proceso de enseñar, como el énfasis que le dan al tipo y tratamiento de los contenidos, las estrategias y métodos de trabajo. Esta relativa autonomía del docente ante la enseñanza, puede entenderse de acuerdo con Ernest (1988) como el resultado de la combinación de tres aspectos:

- Las creencias que tienen los maestros acerca de la enseñanza y el aprendizaje.

- Las limitaciones y oportunidades que ofrece el contexto escolar para la enseñanza.

- La capacidad de los procesos de pensamiento y reflexión de los maestros.

Del reconocimiento de que el trabajo del docente universitario en la educación es decisivo porque son los que llevan a cabo la enseñanza e implementan las orientaciones educativas propuestas por la universidad a través del modelo educativo, se formuló la siguiente pregunta de investigación: ¿Cómo contribuyen los procesos formativos de profesores en ejercicio en la construcción de creencias acerca de la enseñanza en la universidad?

\section{Método}

Esta investigación fue conducida con enfoque cualitativo desde la perspectiva de la teoría fundamentada (Strauss y Corbin, 2016), el diseño metodológico cualitativo fue apropiado para abordar este estudio ya que buscó que fueran los profesores quienes relataran desde su perspectiva, sus experiencias acerca de los procesos formativos durante su ejercicio docente y comprender los significados construidos a partir de esas experiencias, ya que son estos significados lo que interesa indagar dado que representan la realidad socioculturalmente construida (Merriam, 2015). El enfoque de la teoría fundamentada implicó asumir que el objetivo de este tipo de estudios es la construcción de teoría inductiva (Straus y Corbin, 2016). De tal modo que se buscó la representatividad de los conceptos por medio del muestreo teórico y la saturación, al aplicar los procedimientos de comparación constante, codificación abierta, axial y selectiva se logró la generación de teoría emergente.

\section{Descripción de los participantes.}

Participaron diez profesores mexicanos que imparten clases en diferentes áreas del conocimiento en universidades públicas y privadas en Monterrey, N.L. México. Con la intención de encontrar regularidades y variaciones significativas en sus creencias, la selección de los participantes se basó en la representatividad de los conceptos o muestreo teórico y se adoptó el proceso de saturación de los datos (Straus y Corbin, 2016). Para respetar la confidencialidad de los profesores, se siguió la imposición de anonimato sugerido por Gibbs (2014), y se omitió la 
referencia institucional en la cual desarrollan su práctica docente. En la tabla 1 se presentan las características de los profesores.

Tabla 1.

Características de los profesores

\begin{tabular}{|c|c|c|c|c|}
\hline Profesor & $\begin{array}{l}\text { Años en } \\
\text { ejercicio }\end{array}$ & Universidad & Disciplina de enseñanza & Grado de estudios \\
\hline Alberto & 14 & Pública & Administración de operaciones & Doctorado en Administración \\
\hline Alma & 29 & Privada & Química & Maestría en Ciencias \\
\hline Beatriz & 5 & Pública & Sociología de la educación & Maestría en educación \\
\hline Eduardo & 8 & Pública & Propagación de plantas & Maestría en ciencias \\
\hline Elsa & 23 & Privada & Cálculo integral & Maestrías en finanzas \\
\hline Felipe & 25 & Privada & Métodos de investigación & Doctorado en educación \\
\hline Gloria & 24 & Pública & Historia regional de México & Doctorado en educación \\
\hline Jorge & 35 & Privada & $\begin{array}{l}\text { Conversión de energía } \\
\text { electromecánica }\end{array}$ & $\begin{array}{l}\text { Maestría en ingeniería } \\
\text { eléctrica }\end{array}$ \\
\hline Olivia & 22 & Privada & Inglés & $\begin{array}{l}\text { Doctorado en innovación } \\
\text { educativa }\end{array}$ \\
\hline Patricia & 23 & Pública & Investigación en comunicación & Doctorado en educación \\
\hline
\end{tabular}

Fuente: Elaboración propia

La entrevista a profundidad.

Como estrategia de recolección de datos se utilizó la entrevista semiestructurada, ya que es a partir de la propia perspectiva de los profesores como se puede indagar las creencias que están presentes 
en su práctica docente, se sabe que las personas tienden a construir explicaciones causales en torno a sus creencias, y estas se manifiestan a través de declaraciones verbales (Pajares, 1992). Además, esta estrategia es adecuada cuando el asunto de interés ya no está disponible para ser observado y se recurre al relato oral (Lucca y Berríos, 2003; Strauss y Corbin, 2016).

Dado que fue necesario realizar varias entrevistas a cada uno de los participantes con el fin de obtener mayor información, se adoptó la cualidad de entrevista a profundidad (Taylor y Bogdan, 2006). Para obtener narraciones que contuvieran creencias de los profesores acerca de las experiencias formativas se les pidió que relataran acontecimientos o experiencias del pasado y presente, así como sus reacciones ante dichos eventos. Las entrevistas contaron con preguntas generales, de contraste y para ejemplificar (Flores, 2012).

- $\mathrm{Al}$ inicio de la entrevista las preguntas generales partieron de planteamientos amplios o generales.

- Durante la entrevista se hicieron preguntas de contraste y para ejemplificar, las cuales sirvieron para realizar exploraciones más profundas, con este tipo de preguntas se solicitó al participante que diera un ejemplo de un evento o suceso, o en su caso hiciera comparaciones.

- Así también se hicieron recapitulaciones de los relatos emitidos por los participantes y se formularon preguntas específicas para precisar en los mismos.

\section{Procedimiento.}

1. Se contactó a los profesores y se les invitó a participar en la investigación, se les aclaró que la información proporcionada cuidaría en todo momento la confidencialidad. Se informó a los profesores que la investigación requería la realización de varias entrevistas.

2. Se les proporcionó la carta de consentimiento para que dieran su aprobación y firma.

3. Se agendó la cita para la entrevista inicial considerando la disponibilidad de tiempo de los profesores.

4. Se utilizó en la entrevista inicial un guión de entrevista común a todos los profesores.

5. Para recoger la información durante las entrevistas se utilizó grabadora de audio.

Posteriormente, las entrevistas fueron transcritas y revisadas para identificar aspectos o situaciones en las que se requiriera recabar mayor información y de ese modo, preparar el guión para la siguiente entrevista. Cabe señalar que este proceso consideró en particular los relatos de cada uno de los profesores por lo que, los guiones de entrevista fueron distintos para cada uno de los participantes.

\section{Estrategia analítica.}

De acuerdo a Strauss y Corbin (2016) se llevaron a cabo los siguientes procedimientos cualitativos para el análisis emergente de datos: 1) durante la codificación abierta se revisaron las entrevistas de manera reflexiva para identificar regularidades y variaciones para crear categorías pertinentes; 2) la codificación axial consistió en precisar y relacionar las categorías y, 3) La codificación selectiva o proceso en el cual se integraron y refinaron las categorías. Para la construcción de validez se aplicaron las técnicas analíticas de comparación, triangulación de enfoques y confirmación con los participantes, así también en la presentación de resultados se incluyeron citas textuales de los relatos de los profesores, lo cual permitió enriquecer las descripciones, apoyar los hallazgos y dar veracidad (Merriam, 2015; Taylor y Bogdan, 2006; Strauss y Corbin, 2016). 


\section{Resultados}

Los resultados se organizaron en categorías siguiendo los procesos analíticos de la teoría fundamentada, se estableció una categoría selectiva denominada Actualización docente, la cual articula a dos categorías abiertas, denominadas a) Capacitación normativa y conocimiento práctico, y b) Los propios procesos de aprendizaje y las iniciativas personales.

Cada categoría es presentada en tablas con las creencias compartidas por los profesores (regularidades), así como las excepciones (variaciones), sus propiedades o atributos comunes y listas de declaraciones. Así también se describen las características de las categorías acompañadas de citas textuales de los profesores. En la tabla 2 se esquematizan las categorías.

Tabla 2.

Categorías procesos formativos de profesores universitarios

\begin{tabular}{|c|c|c|}
\hline Categoría selectiva & Categorías abiertas & Creencias \\
\hline $\begin{array}{l}\text { Actualización } \\
\text { docente }\end{array}$ & $\begin{array}{l}\text { Los propios procesos de } \\
\text { aprendizaje y las } \\
\text { iniciativas personales. }\end{array}$ & $\begin{array}{l}\text { Énfasis en técnicas didácticas, uso de } \\
\text { tecnología como apoyo a la } \\
\text { implementación del modelo educativo. } \\
\text { Libertad para elegir cursos de } \\
\text { capacitación. } \\
\text { El modelo educativo de universidad se } \\
\text { centra en el alumno, aprendizaje activo o } \\
\text { desarrollo de competencias. } \\
\text { No capacita en el conocimiento de la } \\
\text { disciplina que imparten. } \\
\text { Permite libertad para desarrollar la } \\
\text { enseñanza. } \\
\text { Benigna siempre que no se vea atacada en } \\
\text { sus intereses. } \\
\text { Exigente, de vanguardia, noble. } \\
\text { La propia forma de aprender es } \\
\text { transferible a la enseñanza. } \\
\text { Búsqueda de conocimientos en libros, } \\
\text { investigaciones, casos, problemas como } \\
\text { herramientas para enseñar. } \\
\text { Buscar situaciones de la vida real que } \\
\text { ayuden a contextualizar los } \\
\text { conocimientos. }\end{array}$ \\
\hline
\end{tabular}

Fuente: Elaboración propia 


\section{Capacitación normativa y conocimiento práctico.}

La forma en la cual se estructura la capacitación en la universidad, de acuerdo con las declaraciones de los profesores, juega un papel importante en la definición de la prioridad institucional, la cual consiste en la implementación de un sistema de enseñanza basado en la promoción de habilidades para el manejo de técnicas didácticas, desarrollo de competencias y uso de tecnología aplicables en el aula. Se encontró, además, que la mayoría de los profesores creen tener libertad para elegir la manera de enseñar lo cual asocian con libertad de cátedra, así también la función de universidad se visualiza desprovista de carácter académico. En la tabla 3 se presentan algunas declaraciones de los profesores.

\section{Tabla 3.}

Propiedades y declaraciones de la capacitación normativa y conocimiento práctico

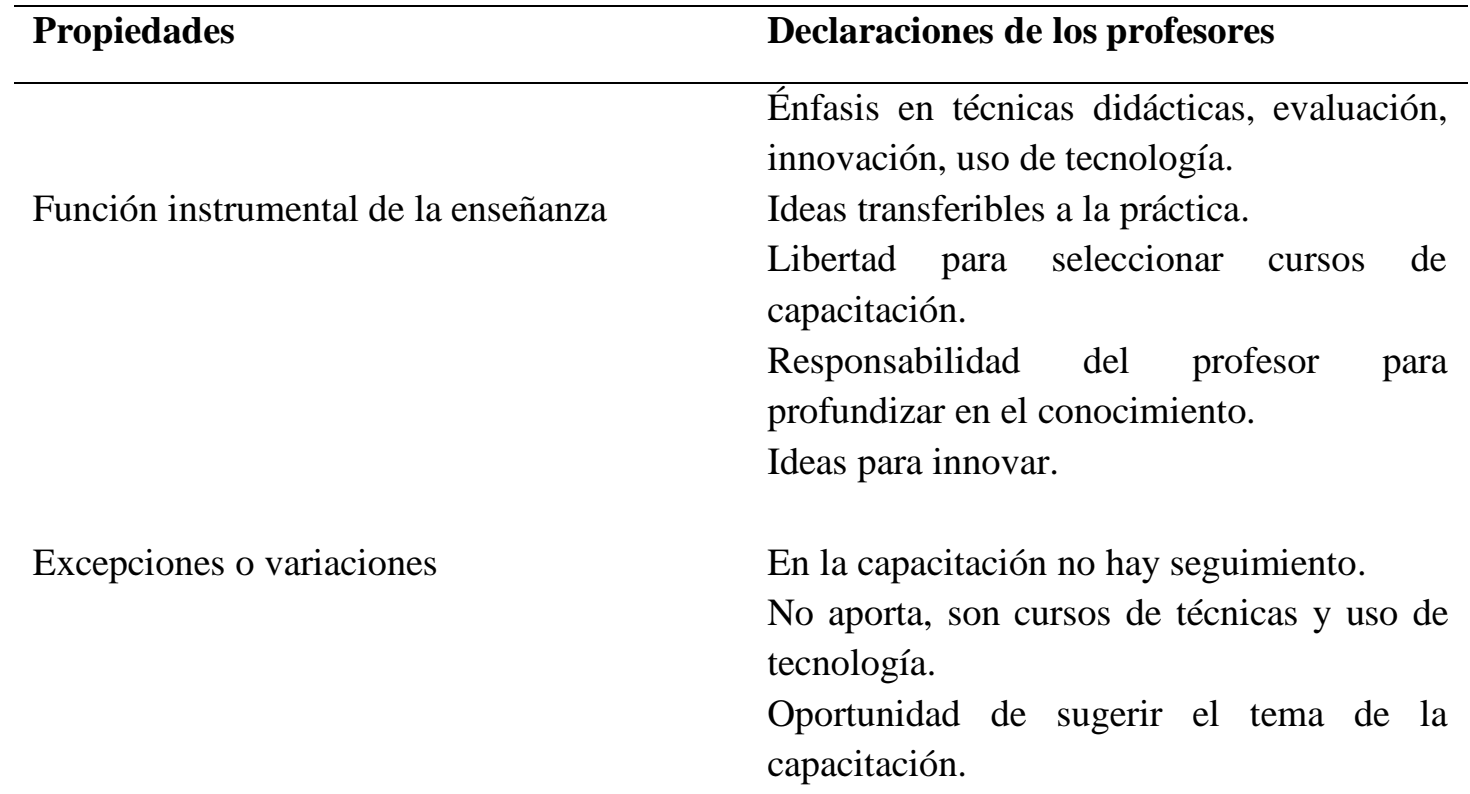

Visión de universidad desprovista de sentido Permite libertad para desarrollar la académico enseñanza.

Benigna siempre que no se vea atacada en sus intereses.

Exigente.

De vanguardia.

Excepciones o variaciones

Relativa libertad para enseñar.

La universidad ejerce control sobre las decisiones del profesor.

Está perdiendo el rumbo de lo que es la educación.

Fuente: Elaboración propia

En relación a los cursos de capacitación que ofrece la universidad todos los profesores creen que el énfasis está relacionado con la actualización en técnicas didácticas, estrategias de 
evaluación y uso de tecnología, lo cual responde al objetivo de incorporar en la práctica el modelo educativo de universidad centrado en el alumno, aprendizaje activo o desarrollo de competencias. Seis profesores creen que la capacitación ha sido importante para la preparación de las clases, se cree que lo que se aprende en las capacitaciones es transferible al diseño de actividades. Esto se logra por medio de un proceso de selección de ideas aplicables en función de la naturaleza del contenido temático de la asignatura que enseñan, la profesora Olivia comentó: "Mucha parte de la capacitación que nos dan aquí yo la he transferido al aula, la institución me da capacitación porque me demanda implementar esas herramientas, por ejemplo, los portafolios, el uso de la plataforma, google docs, google drive”. Por su parte las profesoras Alma y Gloria señalaron:

Yo creo que tomo ideas de las capacitaciones, pues siempre nos han dado mucha capacitación, una capacitación de pensamiento crítico, me interesó mucho esta área y esta, entonces tomo ciertos elementos que yo puedo aplicar en mis clases. Todas las técnicas didácticas, todos los programas, de ahí toma uno ideas, las modificas, las adecuas.

Muy buenos porque hay cursos que ofrece la universidad como institución, pero también hay cursos y conferencias que ofrece la facultad como una dependencia de la universidad. En la universidad hay diplomados en docencia universitaria, hay diplomados en usos de la tecnología, diplomados en tutoría, diplomados en investigación en el caso de la facultad hay generalmente cursos vinculados con la evaluación de programas por competencias, programas sobre el uso de estrategias didácticas.

Como excepciones un profesor mencionó no haber asistido a ninguna capacitación. Tres profesores creen que las capacitaciones no son apropiadas para su práctica docente, las razones que mencionaron fueron que no se enfocan al estudio teórico del área de conocimiento; no hay un seguimiento durante la implementación; están encaminadas a las técnicas didácticas; programas de ética; uso de plataformas y tecnología, al respecto los profesor Felipe dijo "la capacitación es muy relativa, enfocada a la tecnología, a las plataformas, precisamente la capacitación no aporta a mi práctica”. Los profesores Jorge y Beatriz comentaron:

Actualmente no asisto a las capacitaciones, anteriormente si, casi las últimas capacitaciones que se están dando no son propiamente al conocimiento, son técnicas didácticas, los programas de ética, entonces no es propiamente capacitación en el conocimiento, antes si eran, pero cada vez son menos en el área del conocimiento que estamos trabajando.

Lo que sucede es que no son impactantes para mí porque por ejemplo el de competencias se utiliza para hacer los programas del curso, que las redacciones, cumple uno con el curso y hasta ahí, pero en la universidad en sí no hay un seguimiento, a ver maestra ya te di el curso ahora ¿cómo lo estás llevando a cabo, en qué te estás atorando?

En cuanto a la participación de los docentes en sugerir capacitación a partir de sus necesidades e intereses, de los diez profesores participantes uno mencionó que en su facultad se detectan necesidades a través del diálogo con los profesores para organizar cursos y cubrir dicha necesidad: 
Estamos muy en contacto con capacitación para decirle qué es la capacitación que necesitan los profesores porque tenemos plática con ellos, vemos si hay necesidad de implementar simuladores, en las clases se pide esa capacitación para que todos los maestros empiecen a homologar las mismas actividades en sus cursos entonces hay una apertura de solicitar cursos de capacitación específicos para lo que estamos tratando de desarrollar en la academia (Profesor Alberto).

Todos los profesores mencionaron que la universidad deja al profesor la responsabilidad por la actualización en el área del contenido disciplinario. Cinco profesores mencionaron que la actualización en el conocimiento la buscan por su propia cuenta asistiendo a cursos, revisando investigaciones y bibliografía:

No nos dan capacitación en las materias o conocimientos, eso lo tienes que buscar en libros, en artículos (Profesora Alma).

Acerca del conocimiento de la disciplina que ayude no dan ideas de cómo innovar, te dan a conocer las nuevas tendencias educativas, pero hasta ahí (Profesora Elsa).

Cinco profesores señalaron que los cursos de actualización en la disciplina forman parte de un programa de capacitación que ofrecen las facultades y tiene un costo económico, el cual es cubierto por la facultad en la cual trabajan, al respecto el profesor Alberto mencionó "hay una oferta constante de cursos disciplinarios en donde el maestro decide si quiere profundizar, no únicamente en la capacitación interna de la facultad, sino en toda la universidad, incluso puedes ir a cursos internacionales". Otros comentarios respaldan lo anterior:

En lo disciplinario es un tanto más reducido hay que reconocerlo, pero digamos si encontramos es cuestión de buscarlo por ejemplo la asociación de historia tenemos instituida la cátedra, el propósito principal es que vengan investigadores en la disciplina y que nos compartan ya sea a través de una conferencia o algún taller contenidos disciplinarios por ejemplo recientemente vinieron a ofrecer un curso de caligrafía (Profesora Gloria).

En la universidad nos llega por correo la capacitación de todas las facultades, si me interesa me inscribo, claro que en muchos casos corre por tu cuenta, pero te apoyan en la inscripción o depende como anden las cosas. Tiene un costo porque esos cursos los venden las facultades, acá las facultades son autónomas y manejan sus propios recursos (Profesora Patricia).

El vínculo que establecen los profesores con la universidad a través de la capacitación y las creencias implicadas influye en la manera en la que estos profesores la visualizan, ocho profesores mencionaron aspectos tales como que es una institución de vanguardia, exigente, noble, innovadora. Al respecto la profesora Elsa dijo:

Pues es una institución de vanguardia que tiene un alto nivel de exigencia en cuanto a los profesores, que siempre te está invitando a que hagas innovación en el aula. Entonces es algo muy desgastante, pero a la vez te mantiene activo todo el tiempo, las innovaciones vienen acompañadas de capacitaciones que ayudan a la mejora de la práctica docente. 
Es una institución muy noble, muy noble con los profesores porque realmente nos permiten trabajar en la mejora académica de los programas y de cómo impartimos las clases, este se tiene un alto grado de libertad de cátedra sin perder la calidad, yo creo que nos proporcionan las herramientas que necesitamos, la capacitación, los apoyos que necesitamos para desempeñar nuestra labor o sea yo en ese sentido me siento muy bien respaldado (Profesor Alberto).

Como excepciones dos profesores creen que la universidad está cambiando el rumbo de su principal función que es la de preparar a los alumnos dentro de un marco en el cual los profesores estén concentrados en la enseñanza, se pueda profundizar en el conocimiento teórico y el desarrollo del autoaprendizaje en los estudiantes. Al respecto los siguientes comentarios:

No me gusta mucho el camino que está tomando, siento que se está desviando un poquito del objetivo fundamental que es tratar de buscar que el alumno salga bien preparado porque se le está dando importancia a muchos factores que están desviando a muchos profesores de la enseñanza. Ahora se está enfatizando mucho más el que tenga más publicaciones, que la institución salga en cierto nivel del ranking del QS y me da la impresión de que el profesor se desvía un poco de lo que debía ser el trabajo fundamental, tratar de enseñar el material al alumno (Profesor Jorge).

Eso es lo que dice la universidad de sí misma, que siempre está a la vanguardia, quiere parecerse a Harvard, pero ahí el maestro expone en un auditorio y los alumnos estudian, ahí si hay autoaprendizaje. Quisiera que nuestros directores estuvieran frente al grupo de estudiantes para que vieran como son, no saben cuánto es $7 \times 8$, más ahora que los directores son gerentes de empresas que no saben nada de educación (Profesora Alma).

Los profesores comparten la creencia de que la docencia en la universidad se lleva a cabo como un proceso de toma de decisiones en las cuales tienen libertad de elegir la forma de enseñar respetando el programa de estudios, esto es entendido como libertad de cátedra, al respecto las profesoras Beatriz y Patricia dijeron respectivamente "los profesores tenemos libertad, yo diseño mis actividades y el material didáctico", "a mí me dan el programa, tenemos libertad de cátedra porque te dan la temática, pero cómo la manejas ya depende mucho del maestro".

En cuanto al programa de estudios los profesores señalaron que no lo modifican porque se tiene que respetar o porque contiene las temáticas a desarrollar ya que los estudiantes necesitan aprender esos conocimientos para las materias siguientes, la profesora Elsa dijo:

Yo decido el cómo enseñar en cuanto al qué porque ya están los programas oficiales, no puedo agregar un tema o quitar un tema que yo creo no es necesario porque es un programa y se tiene que respetar.

En el programa me dan los temas y los objetivos, si soy muy apegada al temario, porque si existe es que esos temas los van a necesitar en otras materias (Profesora Alma).

Como excepciones dos profesores creen que la libertad en la enseñanza está de alguna manera condicionada o que la universidad ejerce control sobre las decisiones docentes, el profesor Felipe dijo "si he tenido una relativa libertad de cátedra con el contenido mientras no me meta en vericuetos, que no afectes los intereses de la institución". El profesor Jorge señaló: 
Normalmente hay bastante libertad de cátedra, pero cada vez nos ponen más requisitos y como que nos están quitando un poquito eso, por ejemplo, en algunos cursos nos están obligando a que una parte de la calificación les pongamos la participación en el programa in que puede ser conocimiento relacionado con la clase, pero no necesariamente, están quitando un poquito de la decisión del maestro de si acepta eso o no acepta.

Los resultados sugieren que la capacitación docente promueve en los profesores las creencias para el aprendizaje de estrategias, métodos, técnicas didácticas, innovación, uso de la tecnología y conocimiento práctico, lo cual es posible que revele la influencia de la sociedad del conocimiento y la globalización en la orientación educativa de la universidad en la actualidad. Adicional a la capacitación, los profesores recurren por su propia cuenta a otras fuentes que proveen aprendizajes y les ayudan a conducir la enseñanza, esto se describe en la siguiente categoría.

\section{Los propios procesos de aprendizaje y las iniciativas personales.}

En esta categoría se describe un hallazgo no documentado en los estudios de creencias. Se refiere a la forma en la que los profesores aprenden y que utilizan como forma de enseñanza dado que creen en los resultados de su propio aprendizaje. Esta iniciativa personal los conduce a buscar conocimiento, información y maneras de enseñar. Se encontró una relación entre aprender y enseñar al comparar las declaraciones de los profesores cuando se les pidió que describieran cómo estudiaban y la forma en la que preparan las clases.

En el proceso de formación de creencias de acuerdo con Lewis (2002) interviene de manera inevitable el creer en la fuente de lo creído, en este estudio se encontró que los profesores al situarse como sujetos que enseñan, se sitúan también como sujetos que aprenden y creen en sus modos de aprender como una fuente valiosa para transferirlos a la enseñanza. En la tabla 4 se enlistan las declaraciones de los profesores al respecto.

\section{Tabla 4.}

Propiedad y declaraciones de los propios procesos de aprendizaje y las iniciativas personales

Propiedad Declaraciones de los participantes

Escribir frases, conceptos.

Hacer mapas conceptuales, esquemas.

El profesor como sujeto que aprende

Leer, buscar referencias.

Buscar aplicaciones del conocimiento.

Investigación de conocimiento en diversas fuentes.

Buscar situaciones o contextos en los cuales se pueda aplicar el conocimiento.

Cursos, diplomados.

Estudiar.

Excepción o variación

Enseño explicando todo el material, enseño como debí haber estudiado. 
Fuente: Elaboración propia

¿Cómo le hago para que entiendan? es la cuestión principal que se hacen a sí mismos los profesores cuando preparan las clases, los profesores recuperan una serie de estrategias que ellos mismos utilizan para aprender, la siguiente frase describe en términos generales esta categoría "si yo aprendí así, ellos también pueden" (Profesora Olivia), al respecto al profesor Alberto dijo: "Yo aprendí de alguna técnica como la de casos que a mí en lo particular me servía, he incorporado ese método en mi parte docente". Los siguientes comentarios apoyan estos procesos:

Yo no vengo de colegio bilingüe, entonces yo casi tuve que aprender sola, si yo aprendí yo sé cómo se aprendió, entonces me pongo en el lugar de ellos, ;ah! no entienden la voz pasiva, entonces me acuerdo que yo le hice así, trato de ponerles situaciones que digo a mí me funcionó muy bien esto (Profesora Olivia).

A mí se me facilitaba hacer esquemas, así aprendía entonces yo hacia los esquemas, que quede claro el conocimiento teórico, en las clases siempre enseño con esquemas, organizadores, siempre llevo un orden y que haya claridad en el conocimiento (Profesor Felipe).

Yo aprendo en contacto con la práctica, se me dificulta leer y estudiar, por eso se me dificultaba explicar el por qué al enseñar, entonces yo soy más práctico, enseño en la práctica, se aprende ves el árbol, este es un encino (Profesor Eduardo).

Como excepción el profesor Jorge enseña cómo cree que debía haber estudiado cuando fue estudiante universitario, enseña explicando todos los contenidos temáticos para que los alumnos lo comprendan y puedan resolver problemas en cualquier situación, al respecto comentó:

La forma en que me tocó proceder cuando fui estudiantes fue tengo que hacer esta tarea, vamos a ver qué necesito para resolver los problemas de tareas, me iba al libro a buscar la información que se necesitaba para resolverlo, entonces había partes del libro que realmente no me tocaba ver, no se incluía ese material en los problemas que tenía que resolver. Yo enseño explicando para cubrir todo el material y que los alumnos puedan comprender todo lo que viene en el libro.

Todos los profesores creen que revisar y estudiar investigaciones, teoría en artículos científicos o libros, asistir a diplomados y la búsqueda de situaciones de la vida real como contextos para aplicar el conocimiento son los principales aspectos que conducen sus iniciativas en la búsqueda de herramientas para la enseñanza, el profesor Jorge señaló "he preparado mucho este material, haber tenido que recurrir a diferentes libros de texto me ha permitido buscar diferentes formas de presentar el material". Las profesoras Alma y Gloria comentaron respectivamente:

Siempre me pregunto cómo les voy a explicar para que me entiendan esto, siempre he hecho eso, a veces llevo algo experimental, llevo actividades, pero siempre me he preguntado, creo que la clase está diseñada para ellos. Todo el tiempo lo he tenido presente. 
Siempre me pregunto cómo enseñar, he tomado cursos, diplomados, siempre he estado actualizada en lo disciplinario como en lo pedagógico para que los alumnos vean lo valioso que es la enseñanza en historia, donde también se investiga, donde también publicamos, en donde también hacemos una serie de actividades, es decir ser profesora de historia no solamente es reproducir los contenidos de los libros es también generar conocimientos.

El que la propia forma de aprender de los profesores actúe como una fuente valiosa y creíble para planear y conducir la enseñanza, se puede analizar desde dos puntos de vista. Primero, la creencia en los resultados del propio aprendizaje ofrece seguridad y modos de organizar el desarrollo de las clases ante la incertidumbre. Segundo, los datos reflejan que las interacciones entre enseñanza-alumnos se basan en lo que el profesor cree que los alumnos necesitan aprender, estas creencias permiten a los profesores conducir la enseñanza para la comprensión y aplicación del conocimiento y con ello aproximarse al currículum universitario por disciplinas o académico.

\section{Discusión}

Esta investigación se propuso indagar la manera en la que los procesos formativos de profesores en ejercicio contribuyen en la construcción de creencias acerca de la enseñanza en la universidad. Los principales hallazgos indican la creencia de que la capacitación está relacionada con la práctica docente en tanto se trate de cumplir con el modelo educativo de la universidad para que este pueda llegar a implementarse en el salón de clase. Es posible que la capacitación definida institucionalmente y enfocada al aprendizaje de los maestros en docencia universitaria, competencias, habilidades didácticas, uso de recursos educativos y tecnológicos "novedosos o innovadores" haya fomentado en los profesores la creencia de que el modelo educativo se relaciona con el aprendizaje centrado en el alumno, desarrollo de competencias y aprendizaje activo, lo cual indica que estas creencias están sostenidas por una concepción de enseñanza cuyo propósito es que el estudiante desarrolle capacidades para aplicar el conocimiento bajo la conducción y supervisión del docente. Si bien es cierto que este tipo de enseñanza facilita a los estudiantes la comprensión y descubrimiento, en opinión de Joyce, Weil y Calhoun (2012) no es precisamente un modelo centrado en el estudiante dado que lo que se aprende y cómo se aprende se es dado a los alumnos a priori, sin considerar sus intereses y libertad en la elección del qué y cómo aprender, características propias del modelo centrado en el alumno.

En el mismo sentido se encontró la creencia en cuanto a que la universidad enfatiza el saber hacer (procedimental) del profesor al no proporcionar capacitación en el conocimiento de la disciplina, pero sí en habilidades didácticas, esto parece relacionarse con la creencia de que para que se produzca aprendizaje el conocimiento debe ser útil y aplicable, estos hallazgos coinciden con los trabajos críticos de De la Torre (2013), Gimeno (2013), Giroux (2013), Jarvis (2013), Morin (2014) y Ornelas (2009), quienes señalan que valorar el conocimiento solamente por su utilidad es una de las consecuencias negativas derivadas de la sociedad del conocimiento inmersa en la globalización.

En el contexto universitario estudiado los profesores no participan en la definición de la propuesta educativa, en la definición de los contenidos o en los temas de la capacitación, sin embargo, creen en su autonomía en cuanto a la ordenación de los contenidos y en cómo enseñarlo, esto es visto como un espacio de libertad. Los hallazgos revelan que las creencias acerca de la libertad en la toma de decisiones se enmarcan en una cultura en la cual la institución proporciona lineamientos pedagógicos generales, define el contenido a enseñar y orienta la capacitación. Los 
datos sugieren que estas creencias están orientadas por una concepción de la capacitación enfocada a la enseñanza normativa en la cual la prioridad es proporcionar herramientas y alternativas para interesar y provocar aprendizajes en los estudiantes. Lo anterior deja a un lado la función de la capacitación como espacio para el aprendizaje de los profesores en el cual puedan compartir conocimiento, proponer, dialogar, discutir y expresar sus puntos de vista acerca de sus intereses y necesidades intelectuales y pedagógicas, prácticas que apuntan a la concepción del profesor como implementador y diseñador de programas educativos sin participación en la reflexión y crítica curricular (Carbonell, 2015; Díaz Barriga, 2016; Giroux, 2013; Rué Domingo, 2015).

La capacitación es considerada como uno de los espacios educativos que proveen experiencias directas para la adquisición de creencias en acerca de la enseñanza. De acuerdo con investigaciones sobre el tema, para que esta sea relevante en el desarrollo profesional de los profesores debería constituirse en espacios que permitan a los maestros explorar y reflexionar en su práctica, en sus creencias, así como tener como punto de partida sus conocimientos, experiencia, necesidades e intereses (Baptista et al., 2020; Davis y Andrzejewski, 2009; Imbernón, 2002; Kagan, 1992; Murphy y Mason, 2012; Solis, 2015; Woolfolk et al., 2012). Otra consideración respecto a la capacitación docente según LlivinaLavigne (2017) es la preparación de los formadores o capacitadores de docentes, un aspecto poco atendido que ha conducido a que la mayoría tengan pocos conocimientos y experiencia de los problemas que plantea la enseñanza en el aula y de las necesidades de los docentes. Este mismo autor señala que en el caso de las universidades, un aspecto crítico en la preparación de muchos capacitadores está relacionado con el poco protagonismo de los departamentos de educación, como consecuencia de la poca valoración de la profesión docente en la sociedad.

Adicionalmente a la capacitación durante el ejercicio profesional como fuentes de creencias se encontró que los profesores universitarios creen que sus propios modos de aprender son una fuente valiosa de creencias, ya que ellos confían en el proceso y los resultados del propio aprendizaje. Así también declararon tener iniciativas personales que son relevantes en la búsqueda de conocimiento científico y estrategias para planear y conducir la enseñanza. Desde esta mirada, los resultados indican que los profesores pueden crear por sí mismos formas de enseñanza que no aprendieron por la experiencia directa con sus maestros cuando fueron estudiantes y tampoco en la capacitación, lo cual amplia el conocimiento que se tiene acerca de las fuentes de creencias, reportados por Ertmer (2006), Mansour (2009), Nesport (1987), Pajares (1992) y Woolfolk et al. (2012). Aunque los propios procesos de aprendizaje pudiesen considerarse derivados de la experiencia de acuerdo a la clasificación de Lewis (2002), al originarse en la auto experiencia y autorregulación de los profesores vale la pena destacar estas iniciativas personales en una categoría aparte.

Otros estudios también señalan que los profesores debían utilizar el conocimiento de las disciplinas, el pedagógico y de los contextos para desarrollar conocimiento didáctico, así como para analizar y reflexionar en sus prácticas en colaboración (Day y Qing Gu, 2012; Fullan et al., 2016; Hargreaves y Shirley, 2012; Jarauta-Borrasca, Medina-Moya y Mentado-Labao, 2016 y Van Waes, 2018). De acuerdo con los resultados de este estudio, tanto la capacitación como las iniciativas personales y sus prácticas continúan desarrollándose en aislamiento, así también en condiciones institucionales que no ofrecen oportunidades para participar activamente en la discusión y análisis de la propuesta institucional, o en el desarrollo de la enseñanza en conjunto con los estudiantes en un ambiente dialógico, crítico y reflexivo, de este modo la enseñanza se 
orienta más a prácticas influenciadas por la sociedad globalizada, que funcionen para alcanzar resultados competitivos (Giroux, 2013; Jarvis, 2013).

Adicionalmente, los resultados parecen indicar que la universidad al configurarse como institución en la cual las políticas educativas se disemina sin retroalimentación de lo que ocurre en el aula, son condicionantes para que se repitan patrones de enseñanza-aprendizaje. En todo caso la universidad pudiera también aprender al aprovechar los conocimientos, experiencias, compromiso y las iniciativas personales de los docentes para generar una cultura de aprendizaje organizacional, Fullan et al. (2016) señalan que los esfuerzos de cambio e innovación fracasan si no se proporciona a los docentes retroalimentación efectiva y participación en la política educativa para ayudar a dar forma y evaluar las estrategias de mejora.

\section{Referencias}

Baptista, M., Freire, S., \& Freire, A.M. (2020). Science Teachers' Beliefs and Practices: Collaboration as a Trigger of Change. Acta Scientiae, 22(1), 2-22. doi: https://doi.org/10.17648/acta.scientiae.5595

Bingimlas, K., \& Hanrahan, M. (2009). The relationship between teachers' beliefs and their practice: How the literature can inform science education reformers and researchers. En M. F. Tasar \& G. Cakmakci (Eds.), Contemporary Science Education Research: international perspectives (pp. 415-422). Ankara, Turkey: Pegem Akademi.

Caballero, K. (2013). La formación del profesorado universitario y su influencia en el desarrollo de la actividad profesional. Revista de Docencia Universitaria, 11(2), 391-412. Recuperado de http://red-u.net/redu/index.php/REDU/issue/view/71

Carbonell, J. (2015). Pedagogías del siglo XXI. Alternativas para la Innovación educativa. Barcelona, España: Octaedro.

Davis, H., \& Andrzejewski, C. (2009). Teacher Beliefs. En E. Anderman \& L. Anderman. (Eds.). Psychology of classroom learning: An encyclopedia (PCL) (pp. 909-915). New York, NY, EE.UU: Macmillan Reference.

Day, C., y Gu, Q. (2012). Profesores: vidas nuevas, verdades antiguas. una influencia decisiva en la vida de los alumnos. Madrid, España: Narcea.

De la Torre, M. (2013). La universidad, institución milenaria en busca de sentido: la crítica y el humanismo como finalidades universitarias. Autor (Ed.), La Universidad que necesitamos: Reflexiones y debates (pp. 21-48). México: Juan Pablos.

Díaz Barriga, F. (2016). Comprender la teoría del currículum como una conversación complicada: surgimiento, crisis, reconceptualización e internacionalización. Revista mexicana de investigación educativa, 21(69), 641-646. Recuperado de http://www.scielo.org.mx/scielo.php?script=sci_arttext\&pid=S140566662016000200641\&lng=es\&tlng=es.

Ernest, P. (1989). The Knowledge, Beliefs and Attitudes of the Mathematics Teacher: a model. Journal of Education for Teaching: International research and pedagogy, 15 (1), 13-33. doi: 10.1080/0260747890150102

Ertmer, P. A. (2006). Teacher pedagogical beliefs and classroom technology use: A critical link. Trabajo presentado en la American Educational Research Association. Recuperado de https://www.semanticscholar.org/author/Peggy-A.-Ertmer/1688764

Flores-Fahara, M. (2012). Metodología de investigación cualitativa. Monterrey, N.L. Recuperado de editorial digital https://www.editorialdigitaltec.com/ 
Fullan, M., Rincón-Gallardo, S., \& Hargreaves, A. (2015). Professional Capital as Accountability. Education policy analysis archives, 23(15), 1-22. doi: https://doi.org/10.14507/epaa.v23.1998

Gibbs, G. (2014) El análisis de datos cualitativos en investigación cualitativa. Madrid, España: Morata.

Gimeno, J. (2013). En busca del sentido de la educación. Madrid, España: Morata.

Giroux, H. (2013). Pedagogía crítica como proyecto de profecía ejemplar: cultura y política en el nuevo milenio. En Imbernón, F. (Ed.), La educación en el siglo XXI. Los retos del futuro inmediato (pp. 53-62). Barcelona, España: Graó.

Hargreaves, A. y Shirley, D. (2012). La cuarta vía. El prometedor futuro del cambio educativo. Barcelona, España: Octaedro.

Imbernón, F. (2002). Reflexiones globales sobre la formación y desarrollo del profesorado en el Estado Español y Latinoamérica. Revista Educar, 30,15-25. Recuperado de http://www.opech.cl/bibliografico/Doc_Docente/Reflexiones_globlales_sobre_la_forma cion_y_desarrollo_profesional_del_profesorado.pdf

Jarauta Borrasca, B., Medina Moya, J. L., y Mentado Labao, T. (2016). La transformación del saber en la enseñanza universitaria. Una aproximación desde el estudio del CDC. Revista de Investigación Educativa, 34(2), 471-485. doi:10.6018/rie.34.2.221711.

Jarvis, P. (2013). Universidades corporativas: Nuevos modelos de aprendizaje en la sociedad global. Madrid, España: Narcea.

Joyce, B., Weil, M. y Calhoun, E. (2012). Modelos de enseñanza. Barcelona, España: Gedisa.

Kagan, D. M. (1992). Implications of research on teacher belief. Educational Psychologist, 27(1), 65-90.

Lewis, J. (2002). Las seis formas en que elegimos los valores. La cuestión de los valores humanos. Las 6 formas de hacer las elecciones que determinan nuestra vida. Barcelona, España: Gedisa.

Lucca, N., y Berríos, R. (2003). Investigación cualitativa en educación y ciencias sociales. Colombia: Publicaciones Puertorriqueñas, Inc.

Luft, J. A., y Roehring, G. H. (2007). Capturing science teachers' epistemological beliefs: The Development of the Teacher Beliefs Interview. Electronic Journal of Science Education, $\quad 11(2), \quad 38-63 . \quad$ Recuperado de https://ejse.southwestern.edu/article/view/7794

Mansour, N. (2013). Modelling the Sociocultural Contexts of Science Education: The Teachers' Perspective. Research in Science Education, 43, 347-369. doi 10.1007/s11165-0119269-7

Merriam, S. (2015). Qualitative research. A guide to design and implementation. San Francisco, CA: Jossey Bass.

Montes, D. A. y Suárez, C. I. (2016). La formación docente universitaria: claves formativas de universidades españolas Revista Electrónica de Investigación Educativa, 18(3), 51-64. Recuperado de http://redie.uabc.mx/redie/article/view/996

Morin, E. (2014). La vía para el futuro de la humanidad. Barcelona: Paidós.

Murphy, P. K., \& Manson, L. (2012). Changing knowledge and beliefs. En P. A. Alexander \& P. H. Winne (Eds.), Handbook of Educational Psychology (2nd ed) (pp. 305-324). New York: Routledge. doi: 10.4324/9780203874790

Nespor, J. (1987). The role of beliefs in the practice of teaching. Journal of Curriculum Studies, 19(4), 317-328. doi: 10.1080/0022027870190403 
Ornelas, J. (2009). Neoliberalismo y capitalismo académico. En P. Gentili., G. Frigotto., R. Leher y F. Stubrin, (Eds.), Políticas de privatización, espacio público y educación en América Latina (pp. 83-119). Argentina: Homo Sapiens.

Pajares, F. (1992). Teachers' Beliefs and Educational Research: Cleaning Up a Messy Construct. Review of Educational Research. University of Florida, 62(3), 307-32. Disponible: http://rer.sagepub.com/content/62/3/307.short

Pasillas, M. (2011). Profesión y campo disciplinario como marco de orientación y análisis de la práctica docente en el nivel superior de enseñanza. En M de la Torre (Ed.), La docencia universitaria frente al cambio (pp. 53-85). México: Grafo Print.

Pérez, S., y Castaño, R. (2016). Funciones de la Universidad en el siglo XXI: humanística, básica e integral. Revista Electrónica Interuniversitaria de Formación del Profesorado, 19(1), 191-199. doi.org/10.6018/reifop.19.1.202451

Rokeach, M. (1968). Beliefs, attitudes, and values: A theory of organization and change. San Francisco, CA, EE.UU: Jossey-Bass.

Rué Domingo, J. (2015). El desarrollo profesional docente en Educación Superior: agenda, referentes y propuestas para su adopción. REDU. Revista de Docencia Universitaria, 13(3), 217-236. doi: https://doi.org/10.4995/redu.2015.5461.

Strauss, A., y Corbin, J. (2016). Bases de la investigación cualitativa. Técnicas y procedimientos para desarrollar la teoría fundamentada. Colombia: Editorial de la Universidad de Antioquia.

Solis, C. (2015). Creencias sobre enseñanza y aprendizaje en docentes universitarios. Revisión de algunos estudios. Propósitos y Representaciones. 3(2), 227-260. Recuperado de https://revistas.usil.edu.pe/index.php/pyr/article/view/83

Taylor, S.J., y Bogdan, R. (2006). Introducción a los métodos cualitativos de investigación. Madrid, España: Paidós.

Torres, J. (2007). Educación en tiempos de neoliberalismo. Madrid, España: Morata.

UNESCO (2017). La Educación al Servicio de los pueblos y el planeta: creación de futuros sostenibles para todos. Informe de Seguimiento de la Educación en el Mundo. Publicaciones UNESCO (pp. 1-13, 376-385).Recuperado de http://unesdoc.unesco.org/images/0024/002485/248526S.pdf

Van Waes, S., De Maeyer S., Mooleanaar N.M., Van Petegem, P., \& Van den Bosschebd. (2018). Strengthening networks: A social network intervention among higher education teachers. Learning and Instruction. 53, 34-49. doi: https://doi.org/10.1016/j.learninstruc.2017.07.005

Woolfolk, H. A., Davis, H. \& Pape, S. J. (2012). Teacher knowledge and beliefs. En P. A. Alexander \& P. H. Winne (Eds.), Handbook of Educational Psychology (2 nd ed.) (pp. 715- 737). New York: Routledge. doi:10.4324/9780203874790 